\title{
Energy Efficient Capacitive Body Channel Access Schemes for Internet of Bodies
}

\author{
Abeer Alamoudi, Abdulkadir Celik, Ahmed M. Eltawil \\ Computer, Electrical, and Mathematical Sciences and Engineering Division, \\ King Abdullah University of Science and Technology (KAUST), Thuwal, 23955-6900, KSA.
}

\begin{abstract}
The Internet of bodies is a network of wearable, ingestible, injectable, and implantable smart objects located in, on, and around the body. Although radio frequency (RF) systems are considered the default choice for implementing on-body communications, which need to be localized in the vicinity of the human body (typically $<5 \mathrm{~cm}$ ), highly radiative $\mathrm{RF}$ propagations unnecessarily extend several meters beyond the human body. This intuitively degrades energy efficiency, leads to interference and co-existence issues, and exposes sensitive personal data to security threats. As an alternative, the capacitive body channel communication (BCC) couples the signal (between $10 \mathrm{kHz}-100$ MHz) to the human body, which is more conductive than air. Hence, BCC provides a lower propagation loss, better physical layer security, and $\mathbf{n J} / \mathbf{b i t}$ to $\mathrm{pJ} / \mathrm{bit}$ energy efficiency. Accordingly, this paper investigates orthogonal and non-orthogonal capacitive body channel access schemes for ultra-low-power IoB nodes. We present the optimal uplink and downlink power allocations in closed-form, which deliver better fairness and network lifetime than benchmark numerical solvers. For a given bandwidth and data rate requirement, we also derive the maximum affordable number of IoB nodes for both directions of orthogonal and nonorthogonal schemes.
\end{abstract}

Index Terms-Wireless body area networks, capacitive coupling, human body communications, multiple access; power control, ultra-low power, internet of things.

\section{INTRODUCTION}

$\mathrm{T}$ HE Internet of bodies an imminent extension to the vast Internet of Things (IoT) ecosystem, can be defined as a network of uniquely identifiable smart objects in, on, and around the human body [1]. Depending on their operational location, the IoB nodes can be wearable, ingestible, injectable, and implantable devices. This wide variety of IoB nodes could transform our perception of various sectors, such as patient monitoring, preventative healthcare, wellness, fitness, and cybersecurity, to name a few. Noting that IoB has its root in wireless body area networks (WBANs) [2], the IEEE 802.15.6 Standard defines physical and medium-access layer specifications to satisfy various quality of service (QoS) metrics, such as latency, throughput, and power efficiency [3]. To meet these requirements, the IEEE 802.15.6 Standard specifies three main wireless technologies: narrowband (NB) and ultrawideband (UWB) radio frequency (RF) communications and body channel communications (BCC).

The NB and UWB channels experience significantly different attenuation patterns in, on, and around the human body; a lossy, heterogeneous, and frequency-dependent dielectric medium. Even though RF systems have been widely adopted thanks to their own virtues for in-body and off-body links, they are inadequate for on-body communications due to their highly radiative and omnidirectional propagation nature that can cover tens of meters of space around the body. Since on-body communication should occur within the very close vicinity of the body $(<5 \mathrm{~cm})$, radiating $\mathrm{RF}$ signals with such unnecessary coverage 1) reduces energy efficiency, 2) causes interference to/from other IoB nodes, especially in crowded license-free bands, 3) and leads to security risks and privacy concerns as it inadvertently permits eavesdropping and overhearing. Moreover, NB and UWB systems require a complex and power-hungry radio front end, which reduces the node lifetime and needs a larger battery size. Indeed, all of these drawbacks contradict the low-cost, miniature, and ultralow power IoB design goals.

BCC is an alternative form of communication that uses human skin tissues as a transmission medium and confines the signal propagation to the human body. A capacitive BCC is illustrated in Fig. 1, where the signal transmitter and receiver electrodes are in contact with the skin to form the signal (forward) path, whereas the floating ground electrodes form the return (backward) path through the environment. Since the forward path encounters a mild signal fading due to the high body conductivity, the channel attenuation is mainly dominated by over-the-air capacitive backward paths [4].

BCC is specifically designated to operate between $100 \mathrm{kHz}$ and $100 \mathrm{MHz}$ because the body is susceptible to interference below this range, and acts as an antenna above this range. In addition to confining signals to the human body, this operational frequency considerably reduces signal leakage [5], which enhances energy efficiency and physical layer security as a result. Moreover, operating below $100 \mathrm{MHz}$ makes carrierless communication possible, as the transceiver size can be decoupled from the carrier wavelength, eliminating the need for radio front-ends. Indeed, capacitive BCC transceivers have been shown to achieve energy efficiency levels ranging between $\mathrm{nJ} / \mathrm{bit}$ to $\mathrm{pJ} / \mathrm{bit}$ levels [6]-[8]. Overall, the promising BCC technology enables the development of ultra-low-power, miniature, and low-cost IoB devices with better physical layer security attributes.

Recent research efforts have primarily focused on aspects of capacitive BCC channel modelling [1, and references therein] and transceiver design [6]-[8] without sufficiently addressing communication theory and networking prospects. Celik et. al. investigated regular and cooperative orthogonal and non-orthogonal capacitive body channel access schemes in [9], where they consider optimal power and time allocations for max-sum and max-min rate objectives without considering power efficiency aspects. Therefore, this paper proposes energy-efficient orthogonal and non-orthogonal capac- 


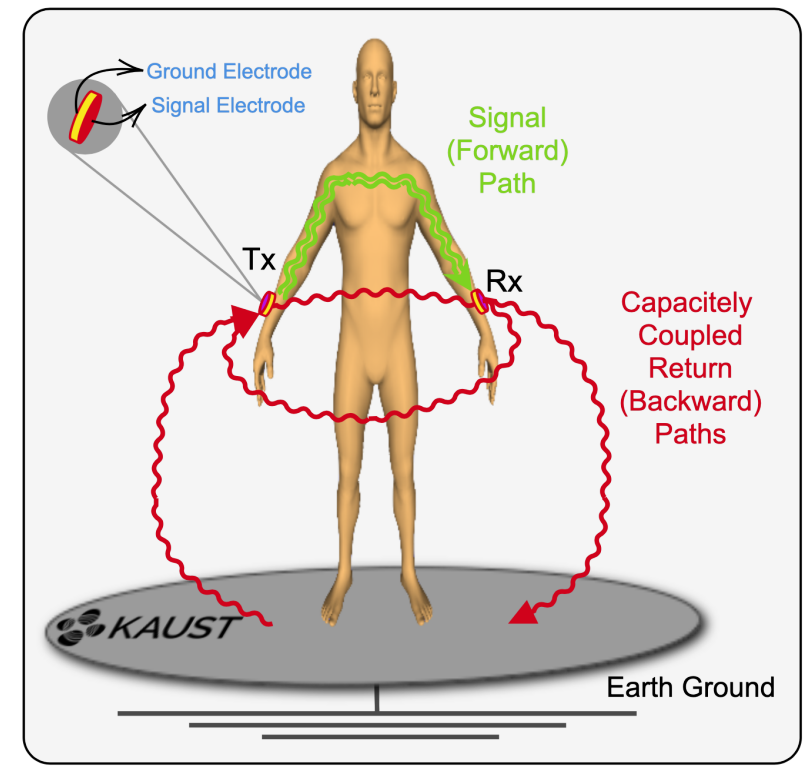

Fig. 1: Illustration of signal and return paths of capacitive body channel communications.

itive body channel access schemes to enable ultra-low-power IoB communications. We present optimal transmission power control in closed-form (CF) for both uplink (UL) and downlink (DL) IoB traffic. Compared to numerical solvers, particularly CVX [10], which is a disciplined convex programming tool, $\mathrm{CF}$ solutions are shown to provide better fairness among IoB nodes. Hence, $\mathrm{CF}$ solutions substantially improve the lifetime of the IoB network. For a given bandwidth and QoS requirement, we also analyze the maximum feasible number of IoB nodes for orthogonal and non-orthogonal schemes in both the UL and DL directions. This analysis is fundamental to unleash the potential of BCC for supporting seamless multinode operation.

The remainder of the paper is organized as follows: Section II presents the capacitive body channel access schemes. Section III formulates the problem and provides closed form solutions for the optimal power control. Section IV analyzes the maximum affordable number of nodes. Section $V$ presents the numerical results. Lastly, Section VI provides the concluding remarks.

\section{Capacitive Body Channel Access}

We consider an IoB network wherein a wearable hub device (e.g., smartwatch) communicates with $K$ on-body IoB nodes through the time-slotted uplink and downlink transmissions. The smartwatch plays the role of an access point that orchestrates the IoB network and exchanges the information with off-body entities (e.g., smartphones, base stations, routers, etc.) utilizing RF communication methods, e.g., cellular, Bluetooth, Wi-Fi, etc. We should note that the proposed methods are not limited to a specific IoB node arrangement over the body, which is a function of the underlying application. Throughout the paper, we denote the total available UL and DL bandwidth for $K$ IoB nodes, time slot duration, and thermal noise power spectral density using $B, T$, and $N_{0}$, respectively. Additionally, $P_{k}$ and $P_{h}$ symbolizes the maximum transmission power of the node and hub, respectively. Without loss of generality, we assume that $P_{k}$ and $P_{h}$ are equal to $P$, ensuring safety compliance with the regulatory bodies. Moreover, the reciprocal channel between $n_{k}$ and $n_{h}$ is represented by $g_{k}^{h}=g_{h}^{h}$. We refer interested readers to [1] for a comprehensive survey of BCC channel models.

\section{A. Orthogonal Multiple Access (OMA)}

The OMA mitigates multiple access interference by allocating each node dedicated and equal bandwidths of $B / K \mathrm{~Hz}$. In the UL transmission, the received signal from the $k^{t h}$ node, $n_{k}$, at the hub node, $n_{h}$, on the $k^{t h}$ subband is given by

$$
y_{k}^{h}=\sqrt{P g_{k}^{h} \dot{\omega}_{k}^{h}} x_{k}+z_{h}, k \in \mathcal{K}
$$

where $\mathcal{K}$ denotes the index set of all IoB nodes, $\dot{\omega}_{k}^{h} \in[0,1]$ is the power allocation weight of the UL-OMA scheme, $x_{k}$ is the transmitted message by the $k^{t h}$ node* $z_{h} \sim \mathcal{N}\left(0, N_{0} B / K\right)$ represents the additive white Gaussian noise at $n_{h}$. Accordingly, the signal-to-noise-ratio (SNR) of UL-OMA is given by $\dot{\gamma}_{k}^{h}=\frac{P g_{k}^{h} \dot{\omega}_{k}^{h}}{N_{0} B / K}$. Therefore, based on Shannon's channel capacity theorem, the maximum UL achievable throughput for $n_{k}$ is given by

$$
\dot{R}_{k}^{h}=\frac{B}{K} \log _{2}\left(1+\dot{\gamma}_{k}^{h}\right), k \in \mathcal{K} .
$$

In DL transmission, the maximum transmission power of $n_{h}$ is also equally divided between nodes, which yields $\dot{\gamma}_{h}^{k}=\frac{P g_{h}^{k} \dot{\omega}_{h}^{k}}{N_{0} B}$. Accordingly, the DL-OMA scheme can easily be obtained by replacing the terms $(\cdot)_{k}$ and $(\cdot)_{k}^{h}$ with the terms $(\cdot)_{h}$ and $(\cdot)_{h}^{k}$ in (1)-(2), respectively.

\section{B. Non-Orthogonal Multiple Access (NOMA)}

Unlike OMA, NOMA permits all IoB nodes to transmit their information concurrently over the entire bandwidth $B$. As a result, the UL and DL receiver nodes $n_{h}$ and $n_{k}$ perform multiuser detection by means of successive interference cancellation (SIC).

1) Uplink NOMA: In UL-NOMA, the observed signal at $n_{h}$ is an aggregation of the transmitted signals, as follows

$$
y_{h}=\sum_{k \in \check{\mathcal{K}}} \sqrt{P g_{k}^{h} \ddot{\omega}_{k}^{h}} x_{k}+z_{h},
$$

where $\check{\mathcal{K}}$ denotes the index set of IoB nodes arranged in ascending order of their channel gains, $\ddot{\omega}_{k}^{h} \in[0,1]$ are the UL-NOMA power allocation weights, and $z_{h} \sim \mathcal{N}\left(0, N_{0} B\right)$. The UL-NOMA scheme allocates power accordingly to ensure that received powers, $p_{k}^{h} \triangleq P g_{k}^{h} \ddot{\omega}_{k}^{h}, \forall k$, can be differentiated and follow the same ascending order, i.e.,

$$
\overbrace{p_{1}^{h}<\ldots<p}^{\text {Interference }} p_{k}^{h} \overbrace{<\ldots<p_{K}^{h}}^{\text {Decodable }}
$$

*We will consider that all transmit messages satisfy $E\left\{\left|x_{k}\right|^{2}\right\}=1, \forall k$, throughout the paper. 
Accordingly, the SIC receiver decodes and subtracts messages in descending order of their received power. In this way, the $n_{h}$ can cancel interference coming from higher rank nodes, while the lower-rank IoB nodes' messages are regarded as interference. The SIC receiver may not cancel all of the interference due to channel estimation errors and hardware limitations. In this case, the signal-to-interference-plus-noise ratio (SINR) of imperfect SIC and the maximum achievable data rate are given by

$$
\ddot{\gamma}_{k}^{h}=\frac{P g_{k}^{h} \ddot{\omega}_{k}^{h}}{\sum_{\substack{i=1 \\ i \in \mathcal{K}}}^{k-1} P g_{i}^{h} \ddot{\omega}_{i}^{h}+\epsilon \sum_{\substack{j=k+1 \\ j \in \check{\mathcal{K}}}}^{K} P g_{j}^{h} \ddot{\omega}_{j}^{h}+N_{0} B},
$$

where the first term in the denominator represents the interference resulting from the decoding order's succeeding messages, whereas the second term constitutes the imperfections in SIC, i.e., $\epsilon \in[0,1]$ is the residual error coefficient. Accordingly, the achievable UL rate of $n_{k}$ is given by

$$
\ddot{R}_{k}^{h}=B \log _{2}\left(1+\ddot{\gamma}_{k}^{h}\right), k \in \check{\mathcal{K}} .
$$

2) Downlink NOMA: In DL-NOMA, $n_{h}$ broadcasts a superimposed signal that is a weighted summation of the intended signals intended for all nodes and received by $n_{k}$ as

$$
y_{k}=\sum_{k \in \hat{\mathcal{K}}} \sqrt{P g_{h}^{k} \ddot{\omega}_{h}^{k}} x_{k}+z_{k},
$$

which is subject to a total power consumption constraint, i.e., $\sum_{k \in \hat{\mathcal{K}}} \ddot{\omega}_{h}^{k} \leqslant 1$ where $\ddot{\omega}_{h}^{k} \in[0,1]$ are the DL-NOMA power allocation weights and $\hat{\mathcal{K}}$ denotes the index set of IoB nodes arranged in descending order of their channel gains. Note that the DL-NOMA follows the opposite decoding order, which yields the following SINR levels for the SIC receiver

$$
\ddot{\gamma}_{h}^{k}=\frac{P g_{h}^{k} \ddot{\omega}_{h}^{k}}{\epsilon \sum_{\substack{i=1 \\ i \in \hat{\mathcal{K}}}}^{k-1} P g_{h}^{i} \ddot{\omega}_{h}^{i}+\sum_{\substack{j=k+1 \\ j \in \hat{\mathcal{K}}}}^{K} P g_{h}^{j} \ddot{\omega}_{h}^{j}+N_{0} B},
$$

Following the SIC procedure, the achievable DL rate for $n_{k}$ is given by

$$
\ddot{R}_{h}^{k}=B \log _{2}\left(1+\ddot{\gamma}_{h}^{k}\right), k \in \hat{\mathcal{K}} .
$$

C. Impacts of Decoding Order on Energy Efficiency, Fairness, and Network Lifetime

The decoding order of SIC receivers has a substantial impact on energy efficiency and power allocation fairness among the nodes. [11]-[13] showed that descending and ascending channel gain ordering deliver the highest NOMA gain over OMA. Noting that these works consider maximum sumthroughput objective, reversing these orders delivers much lower energy consumption while substantially increasing the fairness, which mainly determines the IoB network lifetime. To quantify these key performance metrics, let us first model the power consumption of IoB nodes as follows

$$
P_{k, c}^{u l}=P_{k}^{c r c}+P_{k}^{t x}=E_{k}^{c r c} R_{k}^{h}+P \omega_{k}^{h},
$$

where $P_{k}^{c r c}$ and $P_{k}^{t x}$ are the power consumed by the circuit and transmission. In the BCC transceiver design literature, the energy efficiency of transceivers is often measured using energy consumed per transmitted bit. Therefore, $P_{k}^{c r c}$ can be defined as multiplication of transceiver efficiency, $E_{k}^{c r c}$ [Joules/bit], and the data rate $R_{k}^{h}$ [bps]. On the other hand, effective transmission power is simply weighted maximum

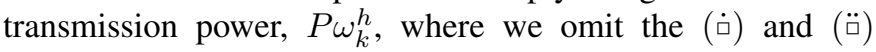
notations to capture both UL-OMA and UL-NOMA schemes. Similarly, the power consumption of the hub is given by

$$
P_{c}^{h}=\sum_{k=1}^{K} P_{k, c}^{d l}=\sum_{k=1}^{K} E_{h}^{c r c} R_{h}^{k}+P \omega_{h}^{k} .
$$

To assess the UL power consumption fairness, we exploit Jain's fairness index, which is defined as follows

$$
\mathcal{J}\left(P_{1, c}^{u l}, P_{2, c}^{u l}, \ldots, P_{K, c}^{u l}\right)=\frac{\left(\sum_{k=1}^{K} P_{k, c}^{u l}\right)^{2}}{K \sum_{k=1}^{K}\left(P_{k, c}^{u l}\right)^{2}} \in[0,1],
$$

can be rewritten for the DL case by replacing $P_{k, c}^{d l}$ with $P_{k, c}^{u l}$. Assuming identical IoB nodes, we define the network lifetime metric as the time span between network initialization and the time slot when the first battery depletion occurred, i.e.,

$$
N L=\min _{\forall k}\left\{\frac{B_{k}^{i n t}}{\alpha_{k} P_{k, c}^{u l} T}\right\},
$$

where $B_{k}^{i n t}$ is the initial battery level and $\alpha_{k}$ is the transmission duty cycle of $n_{k}$. In Section $\mathrm{V}$, we will provide a thorough numerical analysis of these performance metrics.

\section{PRoblem Formulation AND SOlution METHODOLOGY}

\section{A. Problem Formulation}

It is obvious from (13) that the network lifetime is mainly determined by power consumption given in (10) and (11). The optimization problem that optimizes the power allocation weights to minimize total UL power consumption can be formulated as

$$
\begin{array}{ll}
\mathbf{P}_{\mathbf{U L}}: \min _{\mathbf{0} \leq \boldsymbol{\omega} \leq \mathbf{1}} \sum_{k \in \mathcal{K}} P_{c}^{k} \\
\mathrm{C}_{1}: \quad \text { s.t. } \quad R_{k}^{h}(\boldsymbol{\omega}) \geqslant \bar{R}_{k}^{h}, \forall k
\end{array},
$$

where $C_{1}$ is QoS constraints, which ensures that $n_{k}$ is provided with a data rate not less than its demand $\bar{R}_{k}^{h}$ and $\leq$ denotes the pairwise inequality. Similarly, the DL problem can be formulated as

$$
\begin{array}{lll}
\mathbf{P}_{\mathbf{D L}}: \min _{\mathbf{0} \leq \boldsymbol{\omega} \leq \mathbf{1}} & P_{c}^{h} \\
\mathrm{C}_{1}: & \text { s.t. } & R_{h}^{k}(\boldsymbol{\omega}) \geqslant \bar{R}_{h}^{k}, \forall k \\
\mathrm{C}_{2}: & & \sum_{k} \omega_{h}^{k} \leqslant 1
\end{array}
$$

where $C_{2}$ is an additional constraint to ensure the total DL transmission power is less than the maximum transmission power of $n_{h}$. Both (14) and (15) can be formulated as a convex optimization problem and solved by numerical methods (e.g., CVX). However, considering the low-cost and ultra-low-power 
design goals of IoB nodes, it is necessary to derive optimal closed-form $(\mathrm{CF})$ power allocations to reduce hardware cost and power consumption related to the computational complexity.

\section{B. Solution Methodology}

Both $\mathbf{P}_{\mathbf{U L}}$ and $\mathbf{P}_{\text {DL }}$ reach optimums when QoS constraints are active, i.e., satisfy equality, because providing a higherthan-demand data rate increases both circuit and transmission power consumption. Accordingly, these problems can be rewritten as follows

$$
(\mathbf{I}-\boldsymbol{\Gamma} \mathbf{J}) \mathbf{p}=\overline{\boldsymbol{\Gamma}} \boldsymbol{\sigma},
$$

where the vectors are of size $K \times 1$, matrices are of size $K \times K, \mathbf{I}$ is the identity matrix, $\overline{\boldsymbol{\Gamma}}=\operatorname{diag}\left(\bar{\Gamma}_{1}, \ldots, \bar{\Gamma}_{k}, \ldots, \bar{\Gamma}_{K}\right)$ is the diagonal matrix of the SINR thresholds corresponding to QoS demands, $\mathbf{p}$ is the column vector of the received powers, $\boldsymbol{\sigma}$ is the column vector of the receiver noise, and $\mathbf{J}$ is the interference channel gain matrix with entries

$$
J_{i}^{j}= \begin{cases}\text { OMA } & \begin{cases}0, & i<j \\ 0, & i=j, \\ 0, & i>j\end{cases} \\ \text { UL-NOMA }, & \begin{cases}\epsilon, & i<j \\ 0, & i=j, \\ 1, & i>j\end{cases} \\ \text { DL-NOMA }, & \begin{cases}1, & i<j \\ 0, & i=j, \\ \epsilon, & i>j\end{cases} \end{cases}
$$

where entries 1,0 , and $\epsilon$ cases correspond to no interference, cluster-interference, and residual interference, respectively [14]. Note that (16) is subject to $p_{k} \leqslant P \sum_{k} g_{k}^{h}$ due to $\omega_{k}^{h} \leqslant 1$ in the UL. Likewise, in the DL, (16) is also subject to $\sum_{k} p_{k} \leqslant P \sum_{k} g_{k}^{h}$ due to $\sum_{k} \omega_{h}^{k} \leqslant 1$ in addition to $p_{k} \leqslant P \sum_{k} g_{h}^{k}$ due to $\omega_{h}^{k} \leqslant 1$. Assuming that $\epsilon$ cannot be zero in practice, $\mathbf{J}$ has non-negative elements and is generally considered to be irreducible [14]. PerronFrobenius theorem for a non-negative irreducible matrix that the maximum eigenvalue of $\mathbf{J}$ is real-positive. Additionally, the eigenvector corresponding to it is also non-negative [15]. From the standard matrix theory a feasible solution for (16) requires satisfying the necessary and sufficient condition of having the magnitude of the maximum eigenvalue of $\mathbf{H} \triangleq \overline{\boldsymbol{\Gamma}}(\theta) \mathbf{J}$ to be less than unity [14]. Assuming feasible QoS demands, the solution for (16) is then given by $\mathbf{p}^{*}=(\mathbf{I}-\boldsymbol{\Gamma} \mathbf{J})^{-1} \overline{\boldsymbol{\Gamma}} \boldsymbol{\sigma}$, from which the optimal power allocation weights can be obtained as follows:

1) OMA: Defining the SINR constraint using $\gamma_{k}^{h} \triangleq 2^{\frac{\bar{R}_{k}^{h} K}{B}}-$ 1 , the optimal power allocations for UL-OMA and DL-OMA are given by

$$
\begin{aligned}
& \dot{\omega}_{k}^{h, \star}=\frac{\bar{\gamma}_{k}^{h} N_{0} B}{g_{k}^{h} K P}, \forall k \in \mathcal{K}, \text { and } \\
& \dot{\omega}_{h}^{k, \star}=\frac{\bar{\gamma}_{h}^{k} N_{0} B}{g_{h}^{k} P}, \forall k \in \mathcal{K},
\end{aligned}
$$

which are subject to $\dot{\omega}_{k}^{h, \star} \leqslant 1, \forall k$, and $\dot{\omega}_{h}^{k, \star} \leqslant 1, \forall k$, respectively.

2) UL-NOMA: Defining the SINR constraint as $\gamma_{k}^{h} \triangleq$ $2^{\frac{\bar{R}_{k}^{h}}{B}}-1$, the optimal power allocation for the UL-NOMA is given by

$$
\begin{gathered}
\ddot{\omega}_{K}^{h, \star}=\frac{\bar{\gamma}_{K}^{h} \frac{N_{0} B}{P g_{K}^{h}}}{1-\left(\frac{1+\epsilon \gamma_{K}^{h}}{1-\epsilon}\right)\left[1-\left(\frac{1+\epsilon \gamma_{K}^{h}}{1+\gamma_{K}^{h}}\right)^{K-1}\right]} \\
\ddot{\omega}_{k}^{h, \star}=\frac{\ddot{\omega}_{K}^{h, \star}}{P g_{k}^{h}}\left(\frac{1+\epsilon \gamma_{k}^{h}}{1+\gamma_{k}^{h}}\right)^{K-k}, \forall k \in \check{\mathcal{K}} .
\end{gathered}
$$

which can be simplified for the perfect case $(\epsilon \rightarrow 0)$ as follows

$$
\ddot{\omega}_{k}^{h, \star}=\frac{N_{0} B}{P g_{k}^{h}} \gamma_{k}^{h}\left(1+\gamma_{k}^{h}\right)^{k-1}, \forall k \in \check{\mathcal{K}} .
$$

3) DL-NOMA: Defining the SINR constraint using $\gamma_{h}^{k} \triangleq$ $2^{\frac{\bar{R}_{h}^{k}}{B}}-1$, the optimal power allocation for the DL-NOMA is given by

$$
\begin{gathered}
\ddot{\omega}_{h}^{1, \star}=\frac{\gamma_{h}^{1} \frac{N_{0} B}{P g_{h}^{1}}}{1-\left(\frac{1+\epsilon \gamma_{h}^{1}}{1-\epsilon}\right)\left[1-\left(\frac{1+\epsilon \gamma_{h}^{1}}{1+\gamma_{h}^{1}}\right)^{K-1}\right]} \\
\ddot{\omega}_{h}^{k, \star}=\frac{\ddot{\omega}_{h}^{1, \star}}{P g_{h}^{k}}\left(\frac{1+\epsilon \gamma_{h}^{k}}{1+\gamma_{h}^{k}}\right)^{k-1}, \forall k \in \hat{\mathcal{K}} .
\end{gathered}
$$

which can be simplified for the perfect case $(\epsilon \rightarrow 0)$ as follows

$$
\ddot{\omega}_{h}^{k, \star}=\frac{N_{0} B}{P g_{h}^{k}} \gamma_{h}^{k}\left(1+\gamma_{h}^{k}\right)^{K-k}, \forall k \in \hat{\mathcal{K}} .
$$

\section{Maximum Feasible Number of Nodes}

In this section, we obtain the maximum number of nodes, $K_{\text {max }}$, that can be admitted in the IoB system given the total power constraint, throughput requirements, and bandwidth. This is essential to maintain the validity of power control methods.

\section{A. $O M A$}

In the UL-OMA scheme, the optimal power allocation is violated if at least one of the nodes require a weight more than unity as per (18). Since this node is the lowest channel gain user, by intuition, $K_{\max }$ can be obtained from the inequality $\frac{\bar{\gamma}_{k}^{h} N_{0} B}{\bar{g} K P} \leqslant 1$, where $\bar{g}$ is the lowest channel gain in the network. For the sake of analytical tractability, we assume $\gamma_{k}^{h} \gg 1$ and approximate $\gamma_{k}^{h} \triangleq 2^{\frac{\bar{R}_{k}^{h} K}{B}}-1 \approx 2^{\frac{\bar{R}_{k}^{h} K}{B}}$, which yields $K_{\max }$ from the equality above as

$$
K_{\text {max }}=\left\lfloor-\frac{W_{-1}\left(-\frac{N_{0} R}{\bar{g} P} \log (2)\right) B}{\log (2) R}\right\rfloor,
$$

where $W_{-1}(\cdot)$ is the -1 th branch of the Lambert-W function. For the DL-OMA, there is no need for an approximation and $K_{\text {max }}$ can be obtained from $\frac{\bar{\gamma}_{h}^{k} N_{0} B}{\bar{g} P} \leqslant 1$, as follows

$$
K_{\max }=\left\lfloor\frac{B}{R} \log _{2}\left(1+\frac{P \bar{g}}{N_{0} B}\right)\right\rfloor .
$$




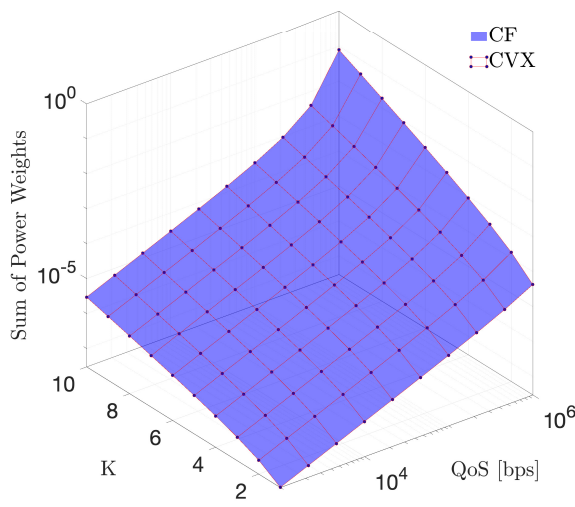

(a)

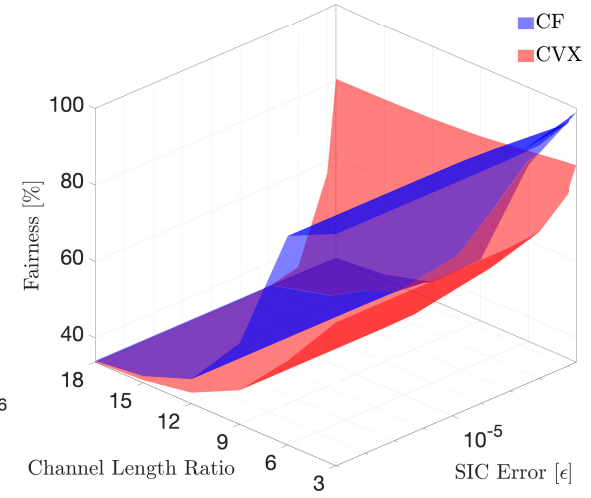

(b)

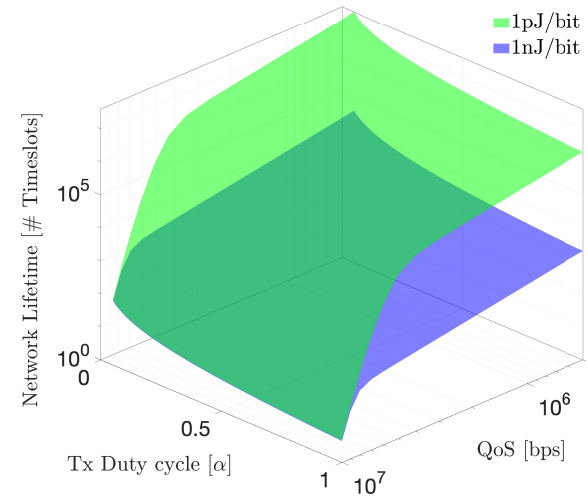

(c)

Fig. 2: Impact of various design parameters on key performance metrics of capacitive BCC: a) sum of power weights obtained using the proposed CF solution and CVX w.r.t QoS and $K$; b) fairness comparison between the proposed CF solutions and CVX w.r.t SIC and channel gain ratio; and c) network lifetime for different transceiver energy efficiencies w.r.t QoS and transmission duty cycle.

\section{B. UL-NOMA}

For the NOMA scheme, we consider perfect SIC and identical QoS requirements, $\bar{R}$, which yields identical SINR thresholds $\bar{\gamma}$. As per (22), the highest channel gain node is allocated the highest transmission power, therefore is expected to violate the power constraint first. Thus, $K_{\max }$ can be obtained from $\frac{N_{0} B}{P \bar{g}} \bar{\gamma}(\bar{\gamma}+1)^{k-1} \leqslant 1$ as follows

$$
K_{\max }=\left\lfloor 1+\frac{\log \left(\frac{P \bar{g}}{\bar{\gamma} N_{0} B}\right)}{\log (1+\bar{\gamma})}\right\rfloor,
$$

where $\bar{g}$ is the maximum channel gain in the IoB network. That is, a node with channel gain $\bar{g}$ can allow up to $K_{\max }-1$ nodes to be admitted in the network.

\section{DL-NOMA}

In contrast to UL-NOMA, the $K_{\max }$ can be derived from the total power weight constraint, $\sum_{k=1}^{K} \omega_{k} \leqslant 1$. As per (22), the highest channel gain node is allocated the highest transmission power, which contributes most to the total weight. By assuming other nodes have the same highest channel gain, $\bar{g}$, the total weight can be approximated as follows

$$
\sum_{k=1}^{K} \omega_{k}=\sum_{k=1}^{K} \rho \bar{\gamma}(\bar{\gamma}+1)^{K} \frac{1}{(\bar{\gamma}+1)^{k}}=\sum_{k=1}^{K} a \varrho^{k}
$$

where $\varrho \triangleq \frac{1}{(\bar{\gamma}+1)}$ and $a=\rho \bar{\gamma} \varrho^{-K}$. By setting $m=1$ and $n=$ $K$ in the geometric progression formula, i.e., $\sum_{k=m}^{n} a \varrho^{k} \triangleq$ $a \frac{\left(\varrho^{m}-\varrho^{n+1}\right)}{1-\varrho},(29)$ can be rewritten as

$$
\sum_{k=1}^{K} \omega_{k}=\frac{\rho \bar{\gamma} \varrho^{-K}\left(\varrho-\varrho^{K+1}\right)}{1-\varrho}=\frac{\rho \bar{\gamma}}{1-\varrho}\left(\varrho^{1-K}-\varrho\right)
$$

By substituting $\varrho \triangleq \frac{1}{(\bar{\gamma}+1)}$ into (30), we obtain $\sum_{k=1}^{K} \omega_{k}=$ $\rho\left[(1+\bar{\gamma})^{K}-1\right] \leqslant 1$, which yields

$$
K_{\max }=\left\lfloor\frac{\log \left(\frac{\rho+1}{\rho}\right)}{\log (1+\bar{\gamma})}\right\rfloor \text {. }
$$

TABLE I: Simulation Parameters

\begin{tabular}{|l|l|l|l|l|l|l|l|}
\hline Par. & Val. & Par. & Val. & Par. & Val. & Par. & Val. \\
\hline \hline $\mathrm{B}$ & $1 \mathrm{MHz}$ & $N_{0}$ & $-174 \mathrm{dBm} / \mathrm{Hz}$ & $B_{k}^{\text {init }}$ & 1 Joules & $K$ & 3 \\
\hline $\mathrm{T}$ & $1 \mathrm{sec}$. & $P_{m}$ & $-30 \mathrm{dBm}$ & $\mathrm{R}$ & $1 \mathrm{Mbps}$ & $\epsilon$ & $1 \mathrm{E}-6$ \\
\hline
\end{tabular}

\section{Numerical RESUlts}

In this section, the results of simulations performed in MATLAB are presented to assess the performance of the proposed energy-efficient body channel access schemes in order to investigate various network design parameters. Unless stated otherwise, the parameters in Table I will be exploited throughout the simulations.

Fig. 2a compares the sum of power weights obtained via the proposed $\mathrm{CF}$ solution and CVX with respect to QoS constraints and the number of IoB nodes. Here, we consider a network that can support up to 10 IoB nodes with equal QoS demands. The nodes are deployed such that the nearest node to the hub is located at $\ell_{1}^{h}=10 \mathrm{~cm}$, and the $k^{\text {th }}$ node is then $k \times 10 \mathrm{~cm}$ away from $n_{h}$. It is clear from Fig. 2a that the results attained by both approaches match tightly, as high QoS demands and larger network size lead to high power consumption.

Fig. $2 \mathrm{~b}$ plots the fairness metric for both CF and CVX as a function of cancellation error $\epsilon$ and relative channel length, in which the latter is modelled as the ratio of channel length for furthest-away node to the closest node i.e., $\ell_{3}^{h} / \ell_{1}^{h}$. We set the channel lengths for the closer nodes in the system as $\ell_{1}^{h}=10$ $\mathrm{cm}$ and $\ell_{2}^{h}=20 \mathrm{~cm}$, while we sweep $\ell_{3}^{h}$ from 30 to $180 \mathrm{~cm}$. As depicted in Fig. 2b, CF performs better than CVX in terms of fair power allocation between IoB nodes. Interestingly, the fairness metric reaches maximum values in both $\mathrm{CF}$ and $\mathrm{CVX}$ as $\epsilon \rightarrow 1$ because $\epsilon=1$ yields an interference network, where all nodes transmit at maximum power without power control. Nonetheless, this fairness does not necessarily imply energy efficiency as $\epsilon=1$ also yields a higher power consumption. Moreover, the impact of the channel length ratio $\ell_{3}^{h} / \ell_{1}^{h}$ at $\epsilon=1$ drastically reduces fairness for CF, but not for CVX. 


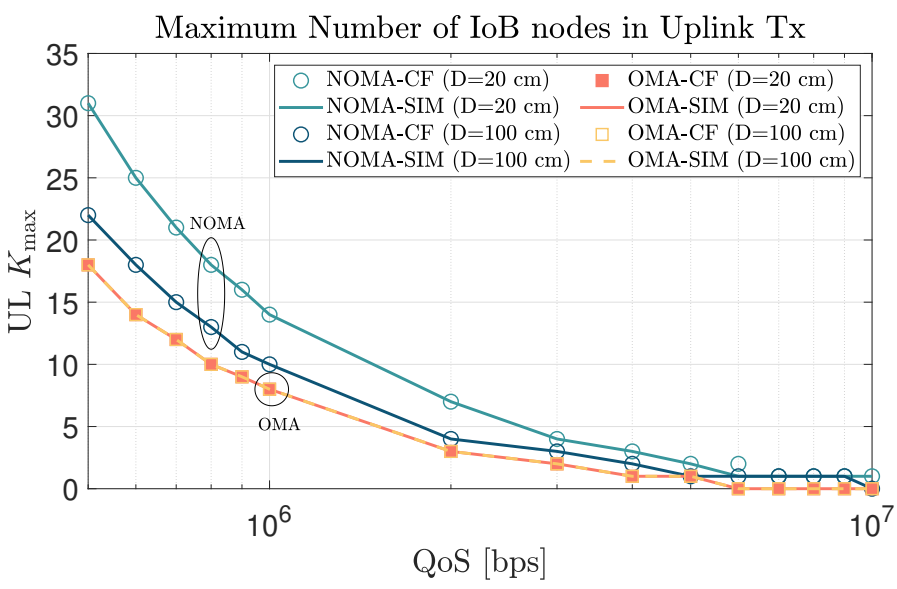

(a)

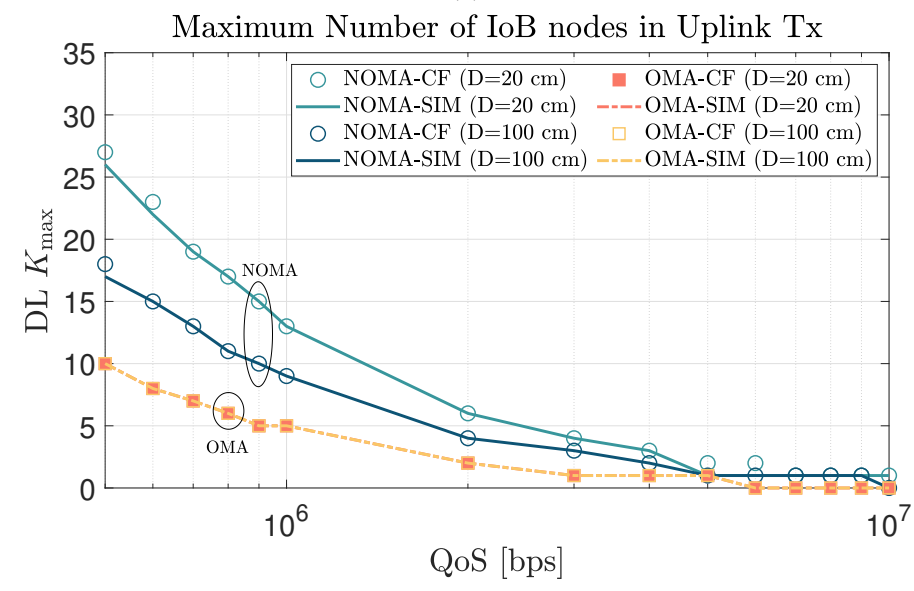

(b)

Fig. 3: $K_{\max }$ vs. QoS: a) UL-OMA vs. UL-NOMA and b) DL-OMA vs. DL-NOMA.

This is because in CVX, all nodes will transmit at maximum power, regardless of their distribution. Proceeding with the $\mathrm{CF}$ optimal power allocation and based on an initial $100 \%$ battery capacity, we plot the network lifetime of identical nodes with respect to the transmission duty cycle and QoS demands in Fig. 2c. Since a considerable amount of energy is consumed by the circuit, we examined two different transceiver energy efficiencies. In both cases, the results agree with the intuition that lower $\alpha$ values (i.e., a lower energy departure rate) and lower QoS demand result in a longer lifespan. Moreover, the overall node lifetime of IoB devices is primarily determined by the circuit's energy efficiency rather than its transmission power. As observed, deploying a transceiver with an energy efficiency of $1 \mathrm{pJ} / \mathrm{bit}$ will increase life compared to $1 \mathrm{~nJ} / \mathrm{bit}$.

In Fig. 3 and Fig. 4, we show how the number of IoB nodes in a network operating on OMA and NOMA access schemes is affected by QoS and maximum transmission power constraints, respectively. Furthermore, in both figures, we illustrate $K_{\max }$ obtained by simulation and close-form equations presented in section IV.Throughout the analysis, we investigate two channel lengths assigned to the nearest node, $\ell_{1}^{h}=20 \mathrm{~cm}$ and

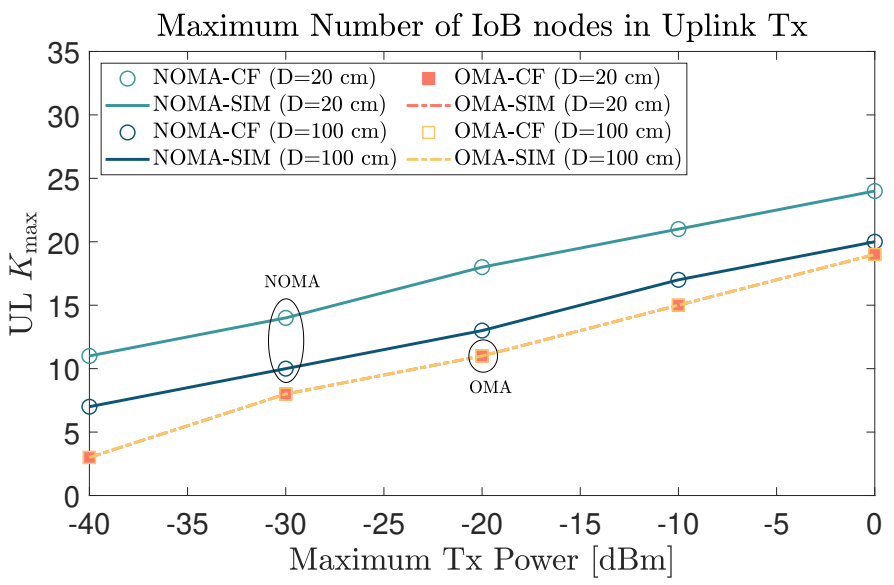

(a)

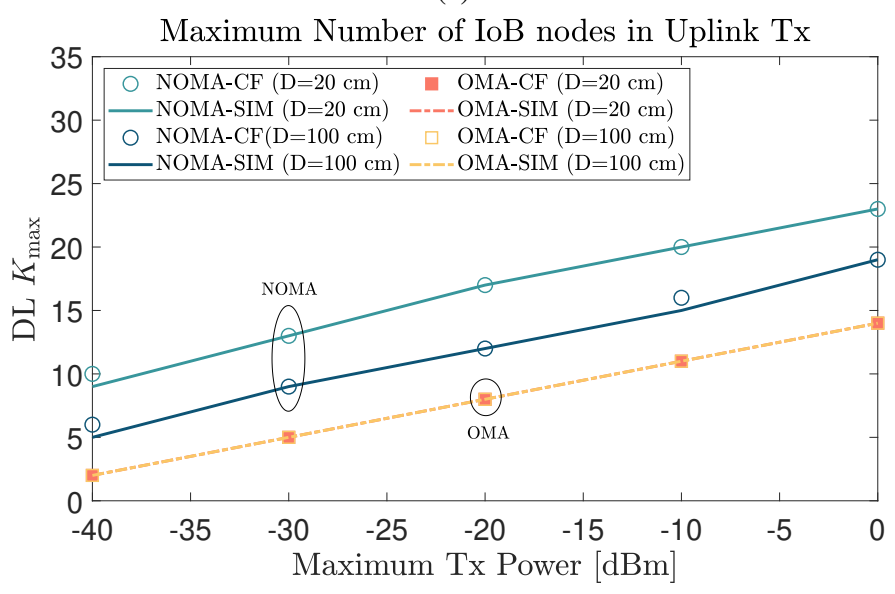

(b)

Fig. 4: $K_{\max }$ vs. Transmission Power: a) UL-OMA vs. UL-NOMA and b) DL-OMA vs. DL-NOMA.

$\ell_{1}^{h}=100 \mathrm{~cm}$, while maintaining the furthest node at $\ell_{K}^{h}=180$ $\mathrm{cm}$ for all cases. Other nodes in the system will follow a uniform distribution, defined as $\ell_{k}^{h}=\ell_{1}^{h}+3(k-1)$. From Fig. 3, we note an inverse relationship between the number of nodes and QoS requirements for both OMA and NOMA in UL and DL traffics. Yet, the most significant observation is that the number of nodes in NOMA is dependent on $\ell_{1}^{h}$, which implies a need to optimize the location of the closest node to the hub. On the contrary, OMA is unaffected by such variations because $K_{\text {max }}$ is governed by $n_{K}$ at $180 \mathrm{~cm}$. Moreover, regardless of the channel length $\ell_{1}^{h}$, the eminence of NOMA over OMA is also evident as NOMA improves the spectral efficiency. In Fig. 4, we see that the number of IoB nodes increases as we increase the maximum transmission power from $-40 \mathrm{dBm}$ to 0 $\mathrm{dBm}$. Similar to Fig. 3, NOMA is dependent on the location of the nearest node to the hub; nonetheless, it still supports more nodes than OMA for all cases.

\section{CONCLUSION}

This paper represents a step forward towards perceiving $\mathrm{BCC}$ from communication and network perspectives, as it 
proposes OMA and NOMA body channel access schemes to facilitate ultra-low power IoB networks. Furthermore, an optimal fair power allocation in closed-form is introduced for both UL and DL transmissions. The simulation results presented confirm the superiority of $\mathrm{CF}$ over $\mathrm{CVX}$, as $\mathrm{CF}$ improves user fairness and prolongs the network lifetime. Additionally, the maximum number of nodes satisfying a feasible and reliable network was derived for OMA and NOMA schemes in both directions. Through simulations, we verified our $K_{\max }$ closed-form equations and elucidated that $K_{\max }$ is correlated with channel length, QoS demands, and maximum transmission power. To address real-life scenarios, we plan to extend our simulations to the impact of non-uniform sensor nodes deployment on the network lifetime. Moreover, we are interested in addressing noise that may arise due to the motion of the body or from biological signals.

\section{REFERENCES}

[1] A. Celik and A. Eltawil, "The internet of bodies: A systematic survey on propagation characterization and channel modeling," IEEE Internet Things J., 2021.

[2] R. Cavallari, F. Martelli, R. Rosini, C. Buratti, and R. Verdone, "A survey on wireless body area networks: Technologies and design challenges," IEEE Commun. Surveys Tuts., vol. 16, no. 3, pp. 1635-1657, Third 2014

[3] "Ieee standard for wireless body area networks," IEEE Std 802.15.62012, pp. 1-271, Feb. 2012.

[4] J. Park, H. Garudadri, and P. P. Mercier, "Channel modeling of miniaturized battery-powered capacitive human body communication systems," IEEE Trans. Biomed. Eng., vol. 64, no. 2, pp. 452-462, 2016.

[5] D. Das, S. Maity, B. Chatterjee, and S. Sen, "Enabling covert body area network using electro-quasistatic human body communication," Scientific reports, vol. 9, no. 1, pp. 1-14, 2019.

[6] S. Maity, B. Chatterjee, G. Chang, and S. Sen, "Bodywire: A 6.3-pj/b 30-mb/s -30-db sir-tolerant broadband interference-robust human body communication transceiver using time domain interference rejection," IEEE Journal of Solid-State Circuits, vol. 54, no. 10, pp. 2892-2906, 2019.

[7] J.-H. Lee, K. Kim, M. Choi, J.-Y. Sim, H.-J. Park, and B. Kim, "A 16.6-pj/b 150-mb/s body channel communication transceiver with decision feedback equalization improving $>200 \%$ area efficiency," in 2017 Symposium on VLSI Circuits. IEEE, 2017, pp. C62-C63.

[8] H. Cho, H. Kim, M. Kim, J. Jang, Y. Lee, K. J. Lee, J. Bae, and H. Yoo, "A $79 \mathrm{pj} / \mathrm{b} 80 \mathrm{mb} / \mathrm{s}$ full-duplex transceiver and a $42.5 \mu \mathrm{W} 100 \mathrm{~kb} / \mathrm{s}$ super-regenerative transceiver for body channel communication," IEEE Journal of Solid-State Circuits, vol. 51, no. 1, pp. 310-317, 2016.

[9] A. Celik and A. Eltawil, "Enabling the internet of bodies through capacitive body channel access schemes," May 2021. [Online]. Available: https://doi.org/10.36227/techrxiv.14535057.v1

[10] M. Grant and S. Boyd, "CVX: Matlab software for disciplined convex programming, version 2.1," http://cvxr.com/cvx, Mar. 2014.

[11] Z. Ding, P. Fan, and H. V. Poor, "Impact of user pairing on 5g nonorthogonal multiple-access downlink transmissions," IEEE Transactions on Vehicular Technology, vol. 65, no. 8, pp. 6010-6023, 2016.

[12] A. Celik, R. M. Radaydeh, F. S. Al-Qahtani, A. H. A. El-Malek, and M. Alouini, "Resource allocation and cluster formation for imperfect NOMA in DL/UL decoupled hetnets," in 2017 IEEE Globecom Workshops, Singapore, December 4-8, 2017. IEEE, 2017, pp. 1-6.

[13] A. Celik, M. Tsai, R. M. Radaydeh, F. S. Al-Qahtani, and M. Alouini, "Distributed user clustering and resource allocation for imperfect NOMA in heterogeneous networks," IEEE Trans. Commun., vol. 67, no. 10, pp. 7211-7227, 2019.

[14] A. Agrawal, J. G. Andrews, J. M. Cioffi, and Teresa Meng, "Iterative power control for imperfect successive interference cancellation," IEEE Transactions on Wireless Communications, vol. 4, no. 3, pp. 878-884, 2005.

[15] R. A. Horn and C. R. Johnson, Matrix analysis. Cambridge university press, 1990. 